\title{
ETIOLOGY FOR LIVER DISEASES IN PEDIATRIC POPULATION
}

\author{
JISMY KARAKKATTU, ROSHNI PR*
}

Department of Pharmacy Practice, Amrita School of Pharmacy, Amrita Vishwa Vidyapeetham University, AIMS Health Sciences Campus, Kochi, Kerala, India. Email: roshnipr@aims.amrita.edu

Received: 15 July 2016, Revised and Accepted: 30 September 2016

ABSTRACT

Objective: The liver diseases affect both the pediatric and adult populations. In the adult population, the stereotype diagnosis in the Indian population is targeted toward males due to excessive alcoholic consumption. Nevertheless, the liver diseases can also affect both the female and pediatric populations. Pediatric liver diseases include cirrhosis, fatty liver diseases, and hepatic failure. The liver diseases are commonly caused by biliary atresia and genetic metabolic diseases. In children, the signs and symptoms of liver diseases are dependent on the principal reason of the liver disease. This review article is to cover all the etiologies that have been identified to cause liver diseases with a special focus on pediatric acute liver failure.

Methods: An extensive PubMed search was conducted and articles that were published after 2007 were included in this article.

Results: The pediatric population etiology of liver diseases can be broadly categorized into infections, immunologic, metabolic, toxin or drug related, indeterminate, and diseases resulting in liver cirrhosis. Complications of pediatric liver diseases include malnutrition, infection, gastroesophageal varices, and hepatic encephalopathy.

Conclusion: Overall, the etiology for liver diseases in the pediatric population is many. Early identification of these factors can improve the quality of life of the pediatric patient. With the correct diagnostic parameters and treatment certain conditions can be completely cured. As for those whose effective treatment is still lacking it is essential to continue the ongoing research until the missing pieces have been identified.

Keywords: Pediatric population, Liver diseases, Acute liver failure, Etiology, Pediatric acute liver failure.

(C) 2017 The Authors. Published by Innovare Academic Sciences Pvt Ltd. This is an open access article under the CC BY license (http://creativecommons org/licenses/by/4. 0/) DOI: http://dx.doi.org/10.22159/ajpcr.2017.v10i1.14121

\section{INTRODUCTION}

Cirrhosis which is found in the majority of liver diseases is process of normal hepatic cells being transformed by fibrosis and nodular regeneration [1]. This eventually leads to the disorganization and malfunction of the liver [1]. The liver diseases are often thought to be limited to adults, specifically males in relation to chronic liver diseases induced by excessive alcohol consumption. However, it is important to remember that pediatric patients are also prone to liver diseases ranging from cirrhosis, fatty liver disease, and even hepatic failure. In infants, cirrhosis is found to be initiated by biliary atresia and genetic metabolic diseases [1]. Cirrhosis in older children results from autoimmune hepatitis, Wilson's disease, alpha-1 antitrypsin deficiency and primary sclerosing cholangitis [1]. Complications of cirrhosis are similar to those found in adults including gastrointestinal (GI) bleeding, ascites, and spontaneous bacterial peritonitis (SBP) [1]. However unlike in adults, pediatric patients with liver diseases are prone to malnutrition and nutritional deficiency disorder. This is because pediatric patients have higher nutritional requirements for growth and development than adults [1]

The most recurrent causes of liver diseases in pediatrics are biliary atresia and inherited syndromes of intrahepatic cholestasis [2]. In the Indian population, liver failure's main cause has found to be due to viral hepatitis [3]. The occurrence of pediatric liver diseases should raise signals as precursors of adult chronic hepatopathies [4]. The liver diseases are often diagnosed late due to the prominent absences of detectable symptoms, especially in the early stages of liver disease [4]. However, in the recent years, new advancements have made way in understanding the pathogenesis of liver diseases [4].

The disease starts with no evident signs or symptoms. As the disease develops, the patients presents liver dysfunction and/or portal hypertension [1]. This is common for both pediatric and adult patients. To predict the status of liver decompensation an increase in hepatic venous pressure gradient is observed [1]. This method is, however, not commonly practice in the clinical setting. The mortality risk is based on calculations using both subjective and objective data in formulas such as the model for end-stage liver disease, pediatric end-stage liver disease, and child-Pugh score [1]. Like with all disease, a detailed history regarding the previous hepatic disease, history of blood or plasma transfusion. In addition, maternal history of any systemic diseases (e.g. hepatitis B or C) should be noted [1].

The signs and symptoms that are presented in the pediatric patient depend on the principal cause of the liver disease. Signs and symptoms of both pediatric and adult patients are similar on many levels. Usually, with pediatric patients, poor weight gain is commonly seen [1]. Observations of spider nevi, visible abdominal circulation, and palmar erythema, telangiectasia of the face and back and repetitive epistaxis is reported [1].

Routine biochemistry parameters should include collection of aminotransferases, canalicular enzymes, bilirubin concentrations, albuminemia, coagulation factors, and prothrombin time. Aminotransferases are known to be the most sensitive indicators of hepatocellular damage [1]. In obstructive liver diseases, it is common to observe elevated levels of alkaline phosphatase and gamma glutamyl transferase, as well as bilirubin concentration [1]. To predict the chances for survival factors that indicate the degree of hepatic synthesis are helpful. Such factors include hypoalbuminemia and deficiency of coagulation factors that are synthesized by the liver [1].

Additional diagnostic techniques include abdominal ultrasound, endoscopy, and liver biopsy. An ultrasound is considered as the ideal 
method for initial investigation of liver diseases [1]. The presence of portal hypertension can be estimated based on the size of the spleen [1] It can also be used in the diagnosis of conditions such as gallstones, choledochal cysts, and Caroli disease [1]. In general, abdominal ultrasounds can be used to identify portal malformations [1]. Endoscopy is ideal for the assessment of the presences, size and extension of gastric, esophageal and even duodenal varices [1]. The gold standard of diagnostic methods for cirrhosis is liver biopsy [1]. Although it is used as a last alternative after all other investigations have been done, it is considered the most accurate. When specimens are taken, it is best that the specimen is evaluated by a pediatric hepatology specialist [1]

Complications of pediatric cirrhosis include malnutrition, infections, gastroesophageal varices and GI bleeding, ascites, SBP, hepatic encephalopathy, acute-on-chronic liver failure, hepatopulmonary syndrome, hepatorenal syndrome, hematological alterations, and hepatocellular carcinoma [1]. The increased energy demands for pediatric patients in addition with anorexia and nausea can obscure the management of malnutrition [1]. Variations in muscle mass and body fat depots indicate the child's nutritional status [1]. Mostly water retention and organomegaly are seen in patients, therefore, using only weight changes in such patients is considered inadequate [1]. Triceps skin fold thickness and upper arm circumference measurements are used to calculate fat and protein reserves. These are used to detect and implement modifications in nutritional status [1].

Cirrhosis can make patients susceptible to infections especially SBP [1] Other commonly observed infections in such patients include urinary tract infections [1]. These can lead to encephalopathy, ascites, and hepatorenal syndrome [1]. The most common cause of GI bleeding is the rupture of gastroesophageal varices [1]. Ascites is also commonly diagnosed in this population [1]. This paper is to discuss the various etiologies that have been found to cause liver diseases in the pediatric population. The rational of this review article is to get an updated view of the current findings that have been published in the available literature.

\section{METHODS}

Identification of articles was conducted using the phrase "etiology of liver diseases in pediatric population" in search engines such as PubMed. Articles that were published before 2007 were excluded from this review article. All articles were reviewed for their relevance and detailed description of various etiological factors and were selected accordingly.

\section{RESULTS}

\section{Pediatric acute liver failure (PALF)}

PALF is known to be the main reason of pediatric liver transplants in the United States annually [5]. Acetaminophen overdose is said to account for fewer than $20 \%$ of PALF cases [6]. The more common causes are metabolic diseases, autoimmune diseases and infectious hepatitis [6] However, it is stated that a diagnosis is not found in up to $50 \%$ of pediatrics [6]. Causes of PALF can be categorized into Table 1.

- Infections

- Immunologic

- Metabolic

- Toxin or drug related

- Indeterminate

- Diseases resulting in liver cirrhosis.

The most common infection to cause PALF is hepatitis A, especially in developing countries. Another common etiology for liver failure is acetaminophen toxicity. Acetaminophen consumption that is acute $(>100 \mathrm{mg} / \mathrm{kg}$ [5]) or chronic is also another known cause of PALF [7]. In chronic consumption of this drug risk factors that further aggravate the condition include concomitant use of other medications that alter hepatic metabolism, delayed medical attention, younger age, and prolonged periods of fasting $[5,8]$. The most common cause of PALF in the pediatric population is claimed to be acetaminophen overdose in the
Table 1: Etiology of PALF; broad classification

\begin{tabular}{|c|c|}
\hline Etiology & Examples \\
\hline Infections & Hepatitis A \\
\hline Immunologic & $\begin{array}{l}\text { Hemophagocytic lymphohistiocytosis, } \\
\text { gestational alloimmune liver disease }\end{array}$ \\
\hline Metabolic & $\begin{array}{l}\text { Galactosemia, tyrosinemia, Niemann-pick } \\
\text { Type C }\end{array}$ \\
\hline Toxin or drug related & Acetaminophen \\
\hline Indeterminate & $\begin{array}{l}\text { Anticonvulsant drugs, antimicrobial agents, } \\
\text { chemotherapeutic agents }\end{array}$ \\
\hline \multirow{4}{*}{$\begin{array}{l}\text { Diseases resulting in } \\
\text { cirrhosis }\end{array}$} & Biliary atresia \\
\hline & Choledochal cysts \\
\hline & Alagille syndrome \\
\hline & Inherited progressive cholestatic syndromes \\
\hline
\end{tabular}

United States and United Kingdom [6]. It is not hepatotoxic at standard therapeutic doses, however, it is a dose-dependent toxin which can results in cell death [6]. The usual pediatric dose is $10-15 \mathrm{mg} / \mathrm{kg} / \mathrm{dose}$ administered every $4 \mathrm{hrs}$ [6]. The maximum dose is $80 \mathrm{mg} / \mathrm{kg} / \mathrm{day}$ [6]. It was also stated that long-term use of drugs such as methotrexate for treatment of rheumatoid arthritis can lead to liver fibrosis as well [9].

A petroleum ether extract of a root (Plumbago zeylanica) was assessed for its hepatoprotective activity for acetaminophen-induced liver damage [10]. Administration of this extract resulted in a significant decrease in serum marks thus indicating a promising protection against acetaminophen induce hepatocellular injury [10]. A hydroalcoholic extract of Cajanus cajan has also shown hepatoprotective activity in carbon tetrachloride induced liver damage [11]. It is claimed that the extract can significantly reduce aspartate aminotransferase, alanine aminotransferase, and increase in the total protein count [11] Perhaps with further studies conducted in the pediatric population these extracts can be used as an aid in the therapeutic management of acetaminophen-induced hepatotoxicity.

Intrinsic hepatotoxins are those with predictable dose-dependent hepatotoxicity. The general principal is that as the dose increases the intensity of hepatotoxicity increases as well usually in a linear function [12]. Examples of such toxicity include amatoxin-containing mushroom poisoning. Consumption of wild mushrooms containing amatoxin is also associated with PALF in children [6]. Ingestion of these types of mushroom is noted in the Western Europe [6]. Therapeutic medications described to cause PALF include amiodarone, isoniazid, minocycline, and pemoline [6].

Idiosyncratic hepatotoxic effects are those drugs that exhibit toxicity that is not predictable or dose-dependent [13]. Common examples of such drugs include isoniazid, propylthiouracil, and halothane [5]. PALF idiosyncratic reactions have also been observed in antimicrobial agents (amoxicillin-clavulanate, minocycline, and azithromycin), anticonvulsant drugs (valproate, phenytoin, carbamazepine, lamotrigine, and felbamate), chemotherapeutic agents (cyclophosphamide, dacarbazine), and other medications (amiodarone and trazodone), etc., as shown in Table 2.

Immune dysregulation is the abnormal response of the immune system [7]. The balance between proinflammatory and antiinflammatory immune responses seems to have a great impact on the prognosis in PALF according to recent studies [8]. The clinical significance of autoimmune markers in patients with PALF is not clear It is understood that natural killer cells play a role in maintaining the balance between proinflammatory and anti-inflammatory responses. Common examples include hemophagocytic lymphohistiocytosis and gestational alloimmune liver disease (neonatal hemochromatosis) [8].

Inherited metabolic disease is metabolic diseases account for at least $10 \%$ of PALF cases [5]. Galactosemia should be considered in a child 
consuming breast milk or other lactose-containing formulas who develops liver failure associated with reducing substances in the urine. Hereditary tyrosinemia Type 1 can present with profound coagulopathy and normal or near normal serum aminotransferase levels. Niemann pick Type $\mathrm{C}$ is a lysosomal storage disease; marked splenomegaly is often noted. Mitochondrial hepatopathies are now increasingly recognized as an important cause of liver failure due to deficiencies in respiratory complexes I, III, or IV or mitochondrial DNA depletion [1].

Older infants and young children presenting with PALF usually have the following metabolic disorders. Mitochondrial hepatopathies especially disorders of fatty acid oxidation [4]. Hereditary fructose intolerance presents only after the introduction of fructose and/or sucrose into an infant's diet and is characterized by recurrent hypoglycemia and vomiting. Argininosuccinate synthetase deficiency and ornithine transcarbamylase deficiency have been associated with PALF although the mechanism of liver injury is uncertain. In older children and adolescents, common metabolic disorders that lead to PALF include the following (Table 3).

\section{Infectious disease}

Hepatitis A, hepatitis B, herpes simplex virus, parvovirus, enterovirus (infants $<7$ months of age), and adenovirus are capable of causing ALF (Table 4).

Infections with viruses other than hepatitis viruses such as the herpes family can cause severe hepatic necrosis [4]. Most commonly affects infants and newborns but can affect all age groups. Epstein-Barr virus is the virus most frequently implicated in PALF older children and adolescents in regions where hepatitis A and B are not endemic [14]. The role and significance of human herpesvirus 6 in ALF is still unclear, however, prevalent in the pediatric population [3,5]. Parvovirus B19 and its association with PLAF still remain unclear. Studies have suggested that the rate of parvovivrus B19 infection among individuals with ALF and aplastic anemia are higher than among patients with ALF to the known causes [5]. In the Indian pediatric population, hepatitis remains the most common cause of liver diseases [3]. Rare causes of PALF can be a presented in conditions such as leukemia, cardiovascular shock associated with systemic hypertension, and celiac disease [6].

Table 2: Drugs known to induced idiosyncratic hepatotoxic effects causing PALF

\begin{tabular}{|c|c|}
\hline Drug class & Examples \\
\hline Anticonvulsant drugs & $\begin{array}{l}\text { Valproate, phenytoin, carbamazepine, } \\
\text { lamotrigine, felbamate }\end{array}$ \\
\hline Antimicrobial agents & $\begin{array}{l}\text { Amoxicillin-clavulanate, minocycline, } \\
\text { azithromycin, roxithromycin, } \\
\text { nitrofurantoin, isoniazid }\end{array}$ \\
\hline Chemotherapeutic agents & Cyclophosphamide, dacarbazine \\
\hline Other & Amiodarone, trazodone \\
\hline Recreational drugs & MDMA \\
\hline $\begin{array}{l}\text { Complementary or } \\
\text { alternative medicines }\end{array}$ & $\begin{array}{l}\text { Pyrrolizidine alkaloids, germander, } \\
\text { chinese herbal medicine, ma huang, } \\
\text { chaparral, black cohosh root, } \\
\text { pennyroyal, kava }\end{array}$ \\
\hline
\end{tabular}

PALF: Pediatric acute liver failure,

MDMA: 3,4-Methylenedioxymethamphetamine

Table 3: Etiology of PALF caused by metabolic disease in older children and adolescents

\begin{tabular}{ll}
\hline Metabolic disease & Age of occurrence \\
Wilson disease & Seen in children over 5 years of age \\
Mitochondrial disease & Occasional present with PALF in \\
& older children and adolescents \\
\hline
\end{tabular}

PALF: Pediatric acute liver failure
Indeterminate causes are seen in about $50 \%$ of pediatrics with PALF [5]. Such cases are identified as intermediate pediatric acute liver failure (I-PALF). Although studies have been conducted to identify novel or unexpected haptotropic viruses in adults, such studies have not been conducted in the pediatric population [5]. I-PALF can be divided into subgroups (Table 5).

Diseases resulting in cirrhosis include biliary atresia and choledochal cysts. Biliary atresia is a condition that arises in the $1^{\text {st }}$ weeks of life. It is categorized by obstruction of the biliary tract. This disease can have two forms; the congenital form and the perinatal form [1]. The fetal form is usually seen at birth in the form of jaundice. In perinatal form, jaundice occurs subsequently the $1^{\text {st }}$ or $2^{\text {nd }}$ week of life. Choledochal cysts are characterized by congenital dilatation of the biliary ducts [1]. It is said to be more common in females than males [1]. The cysts can be grouped into five types: Cystic dilation of the common bile duct, diverticulum of the common bile duct, choledochocele, multiple cysts, and intrahepatic fusiform dilatation.

\section{DISCUSSION}

A review in the World Journal of Hepatology as mentioned that the etiology of PALF can vary according to the patient's age [1]. Other factors that include the prevalence of specific diseases in various regions. However, the majority of the cases consisting of cirrhosis are claimed to be a progression of numerous chronic liver diseases. If the correct diagnostic techniques are used then the presence of cirrhosis, its development and progression can be evaluated and monitored. It is suggested that more research is needed specifically focusing on the pathophysiology of cirrhosis so that its detection and management can be improved.

A review article discussing the current challenges of pediatric liver diseases has stressed the fact that several diseases act as precursors to adult chronic liver diseases such as cirrhosis and hepatocellular carcinoma [7]. Despite many children being admitted in the hospital for pediatric liver diseases the diagnosis is claimed to be underrecognized or discovered via a late diagnosis. Again with further research regarding the pathogenesis of PALF, it can allow physicians to better detect and diagnose the condition in an early stage of the disease.

Table 4: Infectious diseases leading to PALF

\begin{tabular}{ll}
\hline Types of infections & Example \\
\hline Hepatitis virus & A, B \\
Herpes simplex virus & - \\
Epstein-Barr virus & - \\
Human herpesvirus 6 & - \\
Parvovirus B19 & - \\
Other & Adenovirus, dengue fever, members of \\
& enterovirus family, paramyxovirus \\
Non-viral infectious & Infectious agents other than viruses only \\
hepatitis & rarely have been recorded as producing ALF \\
Systemic sepsis & Occasionally presents in a manner that is \\
& virtually indistinguishable from ALF \\
\hline PALF: Pediatric acute liver failure, ALF: Acute liver failure
\end{tabular}

Table 5: I-PALF

\begin{tabular}{ll}
\hline $\begin{array}{l}\text { Sub group } \\
\text { type }\end{array}$ & Description \\
\hline Sub group 1 & $\begin{array}{l}\text { Incomplete diagnostic evaluation due to interruptions } \\
\text { that arise in between planned diagnostic tests, i.e., } \\
\text { Death or clinical improvement } \\
\text { A pathophysiological injury within a patient which is } \\
\text { not identified using current diagnostic strategies }\end{array}$ \\
Sub group
\end{tabular}


A research article published in Indian pediatrics observed 43 patients who were admitted in the pediatric intensive care unit [3]. These patients' etiology and prognostic factors were studied for which 91\% of cases, etiology was confirmed. The study confirmed that the most prevalent etiology of pediatric liver diseases in India is due to viral hepatitis. This was also confirmed in other studies as well [3]. It was suggested that simple interventions such as improving hygiene and immunization coverage can decrease PALF in India. However, this study had a small sample size, therefore, further studies with larger sample sizes representing the general pediatric population are required.

\section{CONCLUSION}

Overall, the etiologies for liver diseases in the pediatric population are many. However, they can be classified broadly into infections, immunologic, metabolic, toxin or drug related, indeterminate, and diseases resulting in liver cirrhosis. Early identification of these factors can improve the quality of life of the pediatric patient. With the correct diagnostic parameters and treatment certain conditions can be completely cured. As for those whose effective treatment are still lacking, it is essential to continue the ongoing research until the missing pieces have been identified.

\section{REFERENCES}

1. Pinto RB, Schneider AC, da Silveira TR. Cirrhosis in children and adolescents: An overview. World J Hepatol 2015;7(3):392-405.

2. Santos JL, Choquette M, Bezerra JA. Cholestatic liver disease in children. Curr Gastroenterol Rep 2010;12(1):30-9.

3. Kaur S, Kumar P, Kumar V, Sarin SK, Kumar A. Etiology and prognostic factors of acute liver failure in children. Indian Pediatr 2013;50(7):677-9.
4. Della Corte C, Mosca A, Vania A, Alterio A, Alisi A, Nobili V. Pediatric liver diseases: Current challenges and future perspectives. Expert Rev Gastroenterol Hepatol 2016;10(2):255-65.

5. Squires RH, Rand EB, Hoppin AG. Acute liver failure in children Etiology and evaluation. Available from: http://www.uptodate.com/ contents/acute-liver-failure-in-children-etiolog-and-evaluation. [Last accessed on 2016 Feb 28].

6. Squires RH Jr. Acute liver failure in children. Semin Liver Dis 2008;28(2):153-66.

7. Leonis MA, Alonso EM, Im K, Belle SH, Squires RH; Pediatric Acute Liver Failure Study Group. Chronic acetaminophen exposure in pediatric acute liver failure. Pediatrics 2013;131(3):e740-6.

8. Reuben A, Koch DG, Lee WM; Acute Liver Failure Study Group. Druginduced acute liver failure: Results of a U.S. Multicenter, prospective study. Hepatology 2010;52(6):2065-76.

9. Shaji A, Joseph S. Low dose methotrexate on long term use in rheumatoid arthritis leads to liver fibrosis - A review. Int J Pharm Res 2013;15:17-21.

10. Kanchana N, Sadiq AM. Hepatoprotective effect of Plumbago zeylanica on paracetamol induced liver toxicity in rats. Int $\mathrm{J}$ Pharm Pharm Sci 2011;3(1):151-4

11. Singh S, Mehta A, Mehta P. Hepatoprotective activity of Canjanus cajan against carbon tetrachloride induced liver damage. Int J Pharm Pharm Sci 2011;3(2):146-7.

12. DiPaola F, Grimley M, Bucuvalas J. Pediatric acute liver failure and immune dysregulation. J Pediatr 2014;164(2):407-9.

13. Molleston JP, Fontana RJ, Lopez MJ, Kleiner DE, Gu J, Chalasani N Drug-Induced Liver Injury Network. Characteristics of idiosyncratic drug-induced liver injury in children: Results from the DILIN prospective study. J Pediatr Gastroenterol Nutr 2011;53(2):182-9.

14. Sundaram SS, Alonso EM, Narkewicz MR, Zhang S, Squires RH; Pediatric Acute Liver Failure Study Group. Characterization and outcomes of young infants with acute liver failure. J Pediatr 2011;159(5):813-818.e1. 